\title{
A general method for proving sharp energy decay rates for memory-dissipative evolution equations
}

\author{
Fatiha Alabau-Boussouira ${ }^{a}$, Piermarco Cannarsa ${ }^{b}$ \\ ${ }^{a}$ L.M.A.M. CNRS-UMR 7122 et INRIA Équipe-projet CORIDA, université Paul-Verlaine-Metz, Ile du Saulcy, 57045 Metz cedex 01, France \\ b Dipartimento di Matematica, Università di Roma Tor Vergata, 00133 Roma, Italy
}

Received 27 November 2008; accepted after revision 15 May 2009

Available online 3 July 2009

Presented by Philippe G. Ciarlet

\begin{abstract}
This Note is concerned with stabilization of hyperbolic systems by a distributed memory feedback. We present here a general method which gives energy decay rates in terms of the asymptotic behavior of the kernel at infinity. This method, which allows us to recover in a natural way the known cases (exponential, polynomial, ...), applies to a large quasi-optimal class of kernels. It also provides sharp energy decay rates compared to the ones that are available in the literature. We give a general condition under which the energy of solutions is shown to decay at least as fast as the kernel at infinity. To cite this article: F. Alabau-Boussouira, P. Cannarsa, C. R. Acad. Sci. Paris, Ser. I 347 (2009).

๑ 2009 Académie des sciences. Published by Elsevier Masson SAS. All rights reserved.
\end{abstract}

\section{Résumé}

Une méthode générale pour obtenir des taux de décroissance de l'énergie des équations d'évolution avec dissipationmémoire. On étudie le problème de la stabilisation des équations de type hyperbolique par un feedback-mémoire distribué. L'objet de cette Note est de montrer qu'il existe une méthode constructive générale qui permet d'obtenir un taux de décroissance de l'énergie en fonction du comportement au voisinage de l'infini du noyau. Cette méthode permet de retrouver de manière naturelle les résultats connus (cas exponentiel, polynômial, ...) mais aussi de définir une classe très générale et quasi-optimale de noyaux à laquelle elle s'applique. Elle permet de montrer sous une condition, aussi très générale, que l'énergie des solutions décroit au moins aussi vite que le noyau à l'infini. Pour citer cet article : F. Alabau-Boussouira, P. Cannarsa, C. R. Acad. Sci. Paris, Ser. I 347 (2009).

(c) 2009 Académie des sciences. Published by Elsevier Masson SAS. All rights reserved.

\section{Version française abrégée}

\section{Introduction}

Cette Note concerne la stabilisation des équations hyperboliques du second ordre par des feedbacks-mémoire distribués. Nous donnons une méthode générale pour l'obtention de taux de décroissance de l'énergie sous des hypothèses

E-mail addresses: alabau@univ-metz.fr (F. Alabau-Boussouira), cannarsa@mat.uniroma2.it (P. Cannarsa). 
très générales sur le noyau de convolution. Cette méthode est constructive et donne des taux de décroissance explicites quand on l'applique à des exemples concrets.

On considère le système (1) où $A: D(A) \subset X \rightarrow X$ est un opérateur linéaire auto-adjoint à domaine dense et vérifiant la condition de coercitivité (2). On rappelle que pour toute fonction continue $k:[0, \infty) \rightarrow(0, \infty)$, décroissante et localement absolument continue vérifiant (3), le problème de Cauchy associé à (1) et de données initiales (4) admet une unique solution faible $u \in \mathcal{C}^{1}([0, T] ; X) \cap \mathcal{C}\left([0, T] ; D\left(A^{1 / 2}\right)\right)$, cf. [18] (et aussi [6]).

On définit l'énergie associée à une solution $u$ de (1) sur $[0, \infty)$ par (13). Etant donnée une solution $u$ de (1)-(4), un problème intéressant est d'étudier le comportement asymptotique de $u$ lorsque $t \rightarrow+\infty$. Ce problème a été exploré dans plusieurs articles sous des conditions différentes sur $k$ (appelée aussi fonction de relaxation), cf. [13,18,17,11,12, $6,3,10,7]$ et les références incluses. Nous considérons des noyaux de convolution vérifiant une condition générale de la forme (7) (où $\chi$ est une fonction strictement positive sur un voisinage à droite et pointé de 0 ). L'un des buts de cette Note est de répondre à la question de savoir si la propriété que l'énergie décroît de la «même façon» que le noyau à l'infini reste vraie dans des cas autres que ceux exponentiels et polynômiaux.

\section{Résultat principal}

On définit l'application $\Lambda$ par (16). Les deux résultats principaux de cette Note sont les suivants :

Théorème 0.1. On suppose que le noyau de convolution $k:[0, \infty) \rightarrow[0, \infty)$ est une fonction localement absolument continue vérifiant (7) où $\chi$ est une fonction donnée, satisfaisant l'hypothèse $(\mathrm{H} 1)$. On suppose de plus que $\chi$ est strictement convexe sur un intervalle $(0, \delta]$ où $\delta>0$ est suffisamment petit et que $\liminf _{x \rightarrow 0^{+}} \Lambda(x)>1 / 2$. Soit u une solution de (1) avec données initiales dans $D\left(A^{1 / 2}\right) \times X$, alors l'énergie $E_{u}$ vérifie l'estimation (17).

Théorème 0.2. On suppose que toutes les hypothèses du Théorème 2.1 sont vérifiées. On suppose de plus que $\limsup _{x \rightarrow 0^{+}} \Lambda(x)<1$ et $k^{\prime}(t)=-\chi(k(t)), t \geqslant 0$ p.p. Alors l'énergie $E_{u}$ vérifie l'estimation (18) et décroit donc au moins aussi vite que $k$ à l'infini.

Remarque 1. - Considérons l'exemple $k(t)=(t+1)^{-1}$ pour $t \geqslant 0$. Alors $\chi(x)=x^{2}$ qui est une fonction strictement convexe. La condition $\int_{0}^{\infty} k(t) \mathrm{d} t<1$ qui garantit le caractère bien posé de (1)-(4) est violée. On a justement $\liminf _{x \rightarrow 0^{+}} \Lambda(x)=1 / 2$ où $\Lambda$ est définie par (16). La condition sur la limite inférieure dans les Théorèmes 2.1 et 2.2 est donc liée à l'intégrabilité de $k$ et donc au caractère bien posé de (1)-(4). Le cas où cette limite inférieure est égale à $1 / 2$ est un cas critique pour lequel le problème peut être bien ou mal posé.

- On peut aussi noter que si $k$ est donnée et strictement décroissante, alors $\chi$ peut n'être connu qu'implicitement. Dans ce cas, la condition (7) doit être remplacée par (12). Notre résultat s'applique encore pourvu que la fonction $\chi$ soit remplacée par la fonction $x \mapsto\left(\varsigma \circ k^{-1}\right)(x) \chi(x)$ (cf. section 3, exemple 3 de la version anglaise pour des détails).

Nous renvoyons à la partie anglaise pour quatre exemples significatifs, deux avec $\chi$ donnée explicitement et deux avec $\chi$ donnée implicitement, ainsi que pour une idée de la preuve.

\section{Introduction}

This Note concerns the stabilization of second order hyperbolic equations by distributed memory-feedbacks. We give a general method to obtain energy decay rates under very general hypotheses on the convolution kernel. Our constructive method gives explicit "optimal" decay rates as well, when applied to concrete examples. We refer the reader to, e.g., $[9,19,15,14,16,1,2]$ frictional feedbacks—a problem that will not be addressed in this Note.

In a real Hilbert space $X$ be, with scalar product $\langle\cdot, \cdot\rangle$ and norm $\|\cdot\|$, let us consider the second order integrodifferential equation

$$
u^{\prime \prime}(t)+A u(t)-\int_{0}^{t} k(t-s) A u(s) \mathrm{d} s=0, \quad t \in(0, \infty),
$$


where $A: D(A) \subset X \rightarrow X$ is a densely defined self-adjoint linear operator satisfying

$$
\langle A x, x\rangle \geqslant \omega\|x\|^{2}, \quad \forall x \in D(A)
$$

for some $\omega>0$. We recall that, for any nonincreasing locally absolutely continuous function $k:[0, \infty) \rightarrow(0, \infty)$ satisfying

$$
\int_{0}^{\infty} k(t) \mathrm{d} t<1,
$$

Eq. (1), complemented with the initial conditions

$$
\left\{\begin{array}{l}
u(0)=u_{0} \in D\left(A^{1 / 2}\right) \\
u^{\prime}(0)=u_{1} \in X
\end{array}\right.
$$

has a unique mild solution $u \in \mathcal{C}^{1}([0, T] ; X) \cap \mathcal{C}\left([0, T] ; D\left(A^{1 / 2}\right)\right)$, see [18] (see also [6]).

Given a solution $u$ of (1)-(4), an interesting problem is to study the asymptotic behavior of $u$ as $t \rightarrow+\infty$. This problem has already been investigated in several papers under different additional assumptions for $k$ (also referred to as the relaxation function), see $[13,18,17,11,12,6,3,10,7]$ and the references therein. For instance, the condition

$$
k^{\prime}(t) \leqslant-\beta k(t) \text { for a.e. } t \geqslant 0
$$

(for some $\beta>0$ ) characterizes exponentially decaying kernels and yields the exponential decay of $u$ at $\infty$. Similarly, the assumption

$$
k^{\prime}(t) \leqslant-\beta k(t)^{1+1 / p} \text { for a.e. } t \geqslant 0
$$

(for some $p \in(1, \infty)$ ) is associated with polynomial type kernels decaying like $(1+t)^{-p}$ and guarantees an analogous behavior of solutions. These two cases are treated, for instance, in [6,17] (see also [8] for coupled Timoshenko beams).

In this Note, we will consider integral kernels satisfying a general condition of the form

$$
k^{\prime}(t) \leqslant-\chi(k(t)) \text { for a.e. } t \geqslant 0
$$

which subsumes (6) and many other interesting cases-the exponential case (5) being well-understood by now. An essential point of our approach is to establish suitable general assumptions for $\chi$, which we do below.

Assumption (H1). $\chi$ is a nonnegative measurable function on $\left[0, k_{0}\right]$, for some $k_{0}>0$, strictly increasing and of class $\mathcal{C}^{1}$ on $\left[0, k_{1}\right]$, for some $k_{1} \in\left(0, k_{0}\right]$, such that

$$
\begin{aligned}
& \chi(0)=\chi^{\prime}(0)=0, \\
& \exists \chi_{0}>0 \text { such that } \chi \geqslant \chi_{0} \text { on }\left[k_{1}, k_{0}\right], \\
& \int_{0}^{k_{0}} \frac{\mathrm{d} x}{\chi(x)}=\infty, \\
& \int_{0}^{k_{0}} \frac{x}{\chi(x)} \mathrm{d} x<1 .
\end{aligned}
$$

Remarks. - Condition (11), together with (7) and the change of variable $x=k(t)$, yields (3) which ensures in turn that (1)-(4) is well-posed. It is important to understand that, unlike the frictional case, the integral condition (3) implies a natural restriction on the growth of the memory damping: $k$ cannot decay too slowly at infinity or, equivalently, $\chi$ cannot be too flat at the origin.

- Notice that, as mentioned above, we have deliberately excluded the case of $\chi^{\prime}(0) \neq 0$, when $k$ decays exponentially at infinity.

- We note that our approach could be useful in situations that look even more general than (7), such as

$$
k^{\prime}(t) \leqslant-\xi(t) \chi(k(t)) \text { for a.e. } t \geqslant 0 \text {. }
$$


Indeed, if $k$ is strictly decreasing-hence, invertible-one can define $\tilde{\chi}(x)=\left(\xi \circ k^{-1}\right)(x) \chi(x)$ and then check if $\tilde{\chi}$ satisfies (H1). This method will be discussed in Example 3 below, for which one can show that the assumptions of Theorem 2.1 are satisfied, even if $\chi$ is known only implicitly.

- Here, we address the context of an abstract second order evolution equation. Thus, our result applies to the wave equation, Petrovsky system, anisotropic elasticity and other models.

Let us define the energy of a given solution $u$ of $(1)$ on $[0, \infty)$ by

$$
E_{u}(t):=\frac{1}{2}\left\|u^{\prime}(t)\right\|^{2}+\frac{1}{2}\left(1-\int_{0}^{t} k(s) \mathrm{d} s\right)\left\|A^{1 / 2} u(t)\right\|^{2}+\frac{1}{2} \int_{0}^{t} k(t-s)\left\|A^{1 / 2} u(s)-A^{1 / 2} u(t)\right\|^{2} \mathrm{~d} s, \quad t \geqslant 0 .
$$

In the above expression, we define the memory-energy of $u$ by

$$
E_{u}^{m}(t)=\int_{0}^{t} k(t-s)\left\|A^{1 / 2} u(s)-A^{1 / 2} u(t)\right\|^{2} \mathrm{~d} s, \quad t \geqslant 0 .
$$

\section{Main results}

The main results of this Note are the following:

Theorem 2.1. Assume that the convolution kernel $k:[0, \infty) \rightarrow[0, \infty)$ is a locally absolutely continuous function such that (7) holds for a given function $\chi$ satisfying hypothesis (H1). Assume, moreover, that $\chi$ is strictly convex on an interval of the form $(0, \delta]$ and

$$
\liminf _{x \rightarrow 0^{+}} \Lambda(x)>\frac{1}{2},
$$

where

$$
\Lambda(x)=\frac{\chi(x) / x}{\chi^{\prime}(x)}, \quad x \in(0, \delta] .
$$

Let $u$ be a solution of (1) with initial data in $D\left(A^{1 / 2}\right) \times X$. Then

$$
E_{u}(t) \leqslant \kappa\left(E_{u}(0)\right) \rho_{1}(t), \quad \forall t \geqslant T_{1},
$$

where $T_{1}>0$ is an explicit universal constant, $\kappa\left(E_{u}(0)\right)$ is a constant which depends on the initial data, and $\rho_{1}$ is a function which decays to 0 as $t \rightarrow \infty$.

Theorem 2.2. In addition to all the assumptions of Theorem 2.1, suppose $\lim \sup _{x \rightarrow 0^{+}} \Lambda(x)<1$ and $k^{\prime}(t)=-\chi(k(t))$ for a.e. $t \geqslant 0$. Then

$$
E_{u}(t) \leqslant \kappa\left(E_{u}(0)\right) k(t), \quad \forall t \geqslant T_{1},
$$

where $T_{1}>0$ and $\kappa\left(E_{u}(0)\right)$ are as above.

Remarks. - Note that the assumption that $\chi$ is convex in a neighborhood $(0, \delta]$ of the origin implies that $\Lambda((0, \delta]) \subset$ $[0,1]$, and $0 \leqslant \liminf _{x \rightarrow 0^{+}} \Lambda(x) \leqslant \limsup _{x \rightarrow 0^{+}} \Lambda(x) \leqslant 1$.

- Consider the example $\chi(x)=x^{2}$ for $x \geqslant 0$, which defines a strictly convex function. Here, condition (11), which guarantees well-posedness of (1)-(4), is violated and one can easily check that $\lim _{x \rightarrow 0^{+}} \Lambda(x)=1 / 2$. The case where the lim inf equals $1 / 2$ in Theorems 2.1 and 2.2 is a critical case for which the problem can be well-posed or ill-posed.

- We note that the conclusion of Theorem 2.2 implies that the energy of $u$ decays at least as fast as the kernel. This kind of optimal decay rate has never been attained at such a level of generality before. On the other hand, if the more restrictive assumptions of such a result are not satisfied but the hypotheses of Theorem 2.1 hold true, that is, if 
$\limsup \operatorname{su}_{x \rightarrow 0^{+}} \Lambda(x)$ is equal to 1 whereas $\liminf _{x \rightarrow 0^{+}} \Lambda(x)>1 / 2$ (see Example 4 below), then the function $\rho_{1}$ in (17) can still be given explicitly in terms of $\chi$. However, due to length considerations, we prefer not to enter into details in the present context. In this case, (17) represents a first step of a more elaborated analysis developed in [5].

An idea of the proof. We first prove that a weighted integral of the total energy can be estimated by the corresponding weighted integral of the memory-energy as follows:

Proposition 2.3. Assume (H1) and let $\phi:[0, \infty) \rightarrow[0, \infty)$ be an absolutely continuous decreasing function. Then, for any $S_{0}>0$, there exist constants $C_{S_{0}}>0$ and $\delta_{4}=\delta_{4}\left(S_{0}\right)>0$ such that, for any $T \geqslant S \geqslant S_{0}$, any solution u of (1) satisfies

$$
\int_{S}^{T} \phi(t) E_{u}(t) \mathrm{d} t \leqslant C_{S_{0}} \phi(S) E_{u}(S)+\delta_{4} \int_{S}^{T} \phi(t)\left(\int_{0}^{t} k(t-s)\left\|A^{1 / 2} u(t)-A^{1 / 2} u(s)\right\|^{2} \mathrm{~d} s\right) \mathrm{d} t,
$$

where $\delta_{4}$ and $C_{S_{0}}$ are independent of $\phi$.

We then prove that we can estimate the memory-energy by introducing sharp weights adapted to the behavior of $\chi$ close to zero (i.e., the behavior of $k$ at infinity), using a general integral inequality theorem and a method introduced for the first time in $[1,2]$. More precisely we show through a pseudo-iterative algorithm that the energy satisfies generalized integral inequalities of the form $\int_{S}^{T} E_{u}(t) F_{i}^{-1}\left(\frac{E_{u}(t)}{\beta_{i}}\right) \mathrm{d} t \leqslant T_{i} E_{u}(S), \forall 0 \leqslant S \leqslant T$, where the indices $i$ vary in a finite subset of the integers, $\beta_{i}$ and $T_{i}$ are positive constants and where the functions $F_{i}$ are invertible (with inverse $F_{i}^{-1}$ ) and satisfy the hypotheses of Theorem 2.3 in Alabau-Boussouira [2]. Applying this theorem, we derive successive energy decay estimates. To compare the decay rate of the solution with that of the kernel, as in Theorem 2.2, one needs the sharper results obtained in [4].

\section{Examples of application}

To illustrate our results, we consider the following three examples:

Example 1: polynomial case. Let $\chi(x)=x^{1+1 / p}, x \in\left[0, k_{0}\right]$, where $p>1$ and $k_{0}>0$ is such that (11) is satisfied.

Example 2: nonpolynomial case. Let $\chi$ be given by $\chi(x)=x^{1+1 / p}(\ln 1 / x)^{-q}$, for $x \in\left[0, k_{0}\right]$ where $p>1, q>0$, and $k_{0}>0$ is such that (11) is satisfied.

Example 3: implicit $\chi$, explicit $k$. Let $k$ be given by $k(t)=\alpha(t+e)^{-p}(\ln (t+e))^{q}$, for $t \geqslant 0$, where $p>1$ and $q>0$ and $\alpha>0$ is chosen such that $\int_{0}^{\infty} k(t) \mathrm{d} t<1$.

Corollary 3.1. The assumptions of Theorem 2.2 are satisfied for the three above examples. Thus the energy of solutions satisfies

$$
E_{u}(t) \leqslant \kappa\left(E_{u}(0)\right) k(t)
$$

for $t$ sufficiently large, and all $\left(u_{0}, u_{1}\right) \in D\left(A^{1 / 2}\right) \times X$. Thus the energies of solutions decay at least as fast as the corresponding kernels for the above three examples.

Note that $\chi$ in Example 3 is not known explicitly. As mentioned before, we can still check that the hypotheses of Theorem 2.2 are satisfied as follows. Differentiating $k$, we derive $\chi(k(t))=-k^{\prime}(t)=k(t)(t+e)^{-1}(p-$ $q \ln (t+e))^{-1}$.

Differentiating once again this expression with respect to $t$, we can show that $\lim _{x \rightarrow 0^{+}} \Lambda(x)=\lim _{t \rightarrow \infty} \Lambda(k(t))=$ $\frac{p}{p+1} \in(1 / 2,1)$. For the above examples we can show that our main result, together with the iterative process to be developed in [5], leads to precise and "optimal" decay rates. In particular, we recover the polynomial case as a special case. These examples are just prototypes and many other significant examples of applications of our results can be 
given. Also, for length considerations we have chosen not to illustrate here our results through examples of concrete PDE's, but the interested reader can consult [6] for such examples.

We furthermore give a fourth example for which $k$ is of sub-exponential growth and the lim sup in Theorem 2.2 is equal to 1 , so that this theorem cannot be applied.

Example 4: implicit $\chi$, explicit $k$. Let $k$ be given by $k(t)=\alpha e^{-\sqrt{t}}$, for $t \geqslant 0$ and where $\alpha>0$ is chosen such that $\int_{0}^{\infty} k(t) \mathrm{d} t<1$. In this case, $\chi$ can be computed explicitly and one can check that $\lim _{x \rightarrow 0^{+}} \Lambda(x)=1$. Hence, the assumptions of Theorem 2.2 are not satisfied, but those of Theorem 2.1 are. So, the energy of the solutions of (1) satisfies, for $t$ sufficiently large,

$$
E_{u}(t) \leqslant \kappa\left(E_{u}(0)\right) \rho_{1}(t), \quad \forall\left(u_{0}, u_{1}\right) \in D\left(A^{1 / 2}\right) \times X,
$$

where $\rho_{1}$ decays to 0 at infinity. This situation is similar to that of exponential decaying kernels: $\rho_{1}$ has the same behavior as $k$ at infinity, but the ratio $E_{u}(t) / k(t)$ is not necessarily bounded (see [5] for a detailed discussion of such a phenomenon).

\section{References}

[1] F. Alabau-Boussouira, Une formule générale pour le taux de décroissance des systèmes dissipatifs non linéaires, C. R. Acad. Sci. Paris, Ser. I 338 (2004) 35-40.

[2] F. Alabau-Boussouira, Convexity and weighted integral inequalities for energy decay rates of nonlinear dissipative hyperbolic systems, Appl. Math. Optim. 51 (1) (2005) 61-105.

[3] F. Alabau-Boussouira, Asymptotic stability of wave equations with memory and frictional boundary dampings, Applicationes Mathematicae 35 (2008) 247-258.

[4] F. Alabau-Boussouira, A unified approach via convexity for optimal energy decay rates of finite and infinite dimensional vibrating damped systems with applications to semi-discretized vibrating damped systems, submitted for publication.

[5] F. Alabau-Boussouira, P. Cannarsa, General decay estimates for memory-damped evolution equations, in preparation.

[6] F. Alabau-Boussouira, P. Cannarsa, D. Sforza, Decay estimates for second order evolution equations with memory, J. Funct. Anal. 254 (2008) $1342-1372$.

[7] F. Alabau-Boussouira, J. Prüss, R. Zacher, Exponential and polynomial stability of a wave equation for boundary memory damping with singular kernels, C. R. Acad. Sci. Paris, Ser. I 347 (2009) 277-282.

[8] F. Ammar-Khodja, A. Benabdallah, J.E. Munoz-Rivera, R. Racke, Energy decay for Timoshenko systems of memory type, J. Differential Equations 194 (1) (2003) 82-115.

[9] C. Bardos, G. Lebeau, J. Rauch, Sharp sufficient conditions for the observation, control, and stabilization of waves from the boundary, SIAM J. Control Optim. 30 (1992) 1024-1065.

[10] P. Cannarsa, D. Sforza, A stability result for a class of nonlinear integrodifferential equations with $L^{1}$ kernels, Applicationes Mathematicae 35 (4) (2008) 395-430.

[11] M.M. Cavalcanti, H.P. Oquendo, Frictional versus viscoelastic damping in a semilinear wave equation, SIAM J. Control Optim. 42 (2003) 1310-1324.

[12] M.M. Cavalcanti, V.N. Domingos Cavalcanti, P. Martinez, General decay rate estimates for viscoelastic dissipative systems, Nonlinear Anal. 68 (2008) 177-193.

[13] C.M. Dafermos, Asymptotic behavior of solutions of evolution equations, Nonlinear evolution equations, Publ. Math. Res. Center Univ. Wisconsin 40, Academic Press, New York, 1978, pp. 103-123.

[14] V. Komornik, Exact Controllability and Stabilization, The Multiplier Method, Collection RMA, vol. 36, Masson-John Wiley, Paris-Chichester, 1994.

[15] I. Lasiecka, D. Tataru, Uniform boundary stabilization of semilinear wave equation with nonlinear boundary damping, Differential Integral Equations 8 (1993) 507-533.

[16] G. Lebeau, E. Zuazua, Decay rates for the three-dimensional linear system of thermoelasticity, Arch. Ration. Mech. Anal. 148 (1999) 179-231.

[17] J.E. Muñoz Rivera, A. Peres Salvatierra, Asymptotic behavior of the energy in partially viscoelastic materials, Quart. Appl. Math. 59 (2001) 557-578.

[18] J. Prüss, Evolutionary Integral Equations and Applications, Monographs in Mathematics, vol. 87, Birkhäuser Verlag, Basel, 1993.

[19] E. Zuazua, Exponential decay for the semilinear wave equation with locally distributed damping, Comm. Partial Differential Equations 15 (1990) 205-235. 